Portland State University

PDXScholar

8-1-1989

\title{
Temporal patterns of nectar and pollen production in Aralia hispida: implications for reproductive success
}

James D. Thomas

Mary A. McKenna

Mitchell B. Cruzan

Portland State University

Follow this and additional works at: https://pdxscholar.library.pdx.edu/bio_fac

Part of the Biology Commons, and the Plant Biology Commons

Let us know how access to this document benefits you.

\section{Citation Details}

Thomson, J. D., Cruzan, M. B., and McKenna, M. A. (1989). Temporal patterns of nectar and pollen production in Aralia hispida: implications for reproductive success. Ecology : A Publication of The Ecological Society of America, 70(4), 1061-1068.

This Article is brought to you for free and open access. It has been accepted for inclusion in Biology Faculty Publications and Presentations by an authorized administrator of PDXScholar. Please contact us if we can make this document more accessible: pdxscholar@pdx.edu. 


\title{
TEMPORAL PATTERNS OF NECTAR AND POLLEN PRODUCTION IN ARALIA HISPIDA: IMPLICATIONS FOR REPRODUCTIVE SUCCESS ${ }^{1}$
}

\author{
James D. Thomson, Mary A. McKenna, ${ }^{2}$ And Mitchell B. Cruzan \\ Department of Ecology and Evolution, State University of New York, \\ Stony Brook, New York 11794 USA
}

\begin{abstract}
Large plants of Aralia hispida present their pollen and nectar in hundreds of small flowers that open sequentially over 2-3 wk in a pattern of synchronized protandry that alternates male and female phases. The primary pollinators, bumble bees, are able to discover individual plants with elevated levels of either nectar or pollen, and to return to them more often than to less rewarding plants. Both pollen and nectar are presented gradually over time in such a way as to favor traplining behavior by the bees, with many, frequent visits. In controlled environments, lifetime sugar production per flower varies among plants, among umbel orders within plants, and between male and female phases of bloom within umbel orders; there are further interactions among these sources of variation, which complicate any consideration of differential male-phase vs. female-phase nectar production. After draining, floral nectar is rapidly replenished, rendering umbels attractive to bees again within $15 \mathrm{~min}$. Pollen production per flower and pollen grain size vary among umbel orders, increasing toward the end of bloom; grain size (but not production) varies among plants as well. Given the high visitation rate by bees, the gradual presentation of pollen should result in more efficient pollen dispersal than simultaneous presentation would.
\end{abstract}

Key words: andromonoecy; anther dehiscence schedule; Aralia hispida; nectar; pollen; pollen size; pollination success; trapline foraging.

\section{INTRODUCTION}

Other work on the reproductive biology and pollination ecology of Aralia hispida has shown that bumble bees, the primary pollinators, respond to experimentally induced variation in inflorescence size and nectar and pollen amounts (Thomson 1988, 1989). This paper (1) describes the temporal patterns of nectar and pollen production within plants, and (2) interprets the consequences of such variation for the mating system of the plants (Thomson and Barrett 1981a), in light of the previous work on bee behavior (Thomson et al. 1982, 1987).

\section{Background: sex expression and bee behavior}

Aralia hispida plants are andromonoecious, typically producing numerous, small, greenish-white flowers in three ranks (primary, secondary, tertiary) of umbel orders. A single primary umbel is subtended by a number of secondaries, which are in turn subtended by tertiaries. Within an order, all umbels bloom synchronously, and all flowers of one order open before any flowers of the next order start. Umbel orders further show synchronized protandry at the level of the plant:

${ }^{1}$ Manuscript received 16 May 1988: revised 24 September 1988: accepted 6 October 1988.

2 Present address: Department of Botany, Howard University, Washington, D.C. 20059 USA. all flowers open first as males, shedding pollen from five anthers (Fig. 1A). After 5-7 d of gradual flower openings, all petals and anthers abscise and, in some flowers only, five styles elongate and diverge, signalling the onset of female function (Fig. 1B). Ramets within clones tend to undergo these changes synchronously. Flowers secrete nectar in both phases. In principle, stigmas remain receptive long enough (4-6 d) to be pollinated geitonogamously by the male flowers of the succeeding umbel order, but the insect visitation rates are so high that most female-phase flowers are crosspollinated prior to the opening of the flowers of the next umbel order (Thomson and Barrett 1981a).

Although many insects visit $A$. hispida, Bombus spp. workers predominate numerically and greatly exceed other insects in numbers of visits and quantity of pollen moved. The more common Bombus species (perhaps all of them) trapline the plants they visit, restricting their foraging to small areas $\left(\approx 100 \mathrm{~m}^{2}\right.$ in dense stands), and tending to visit a particular set of flowering shoots in a regular sequence (Thomson et al. 1982). This sequence will be repeated several times during one foraging trip, resulting in revisitation intervals as low as $10 \mathrm{~min}$. Bees actively collect both pollen and nectar, and strongly prefer male-phase umbels to female ones in choice tests. Bees do sample shoots other than their primary set, and will add or drop plants from their traplines, gradually shifting their traplines to include more rewarding plants (Thomson et al. 1987). Manip- 

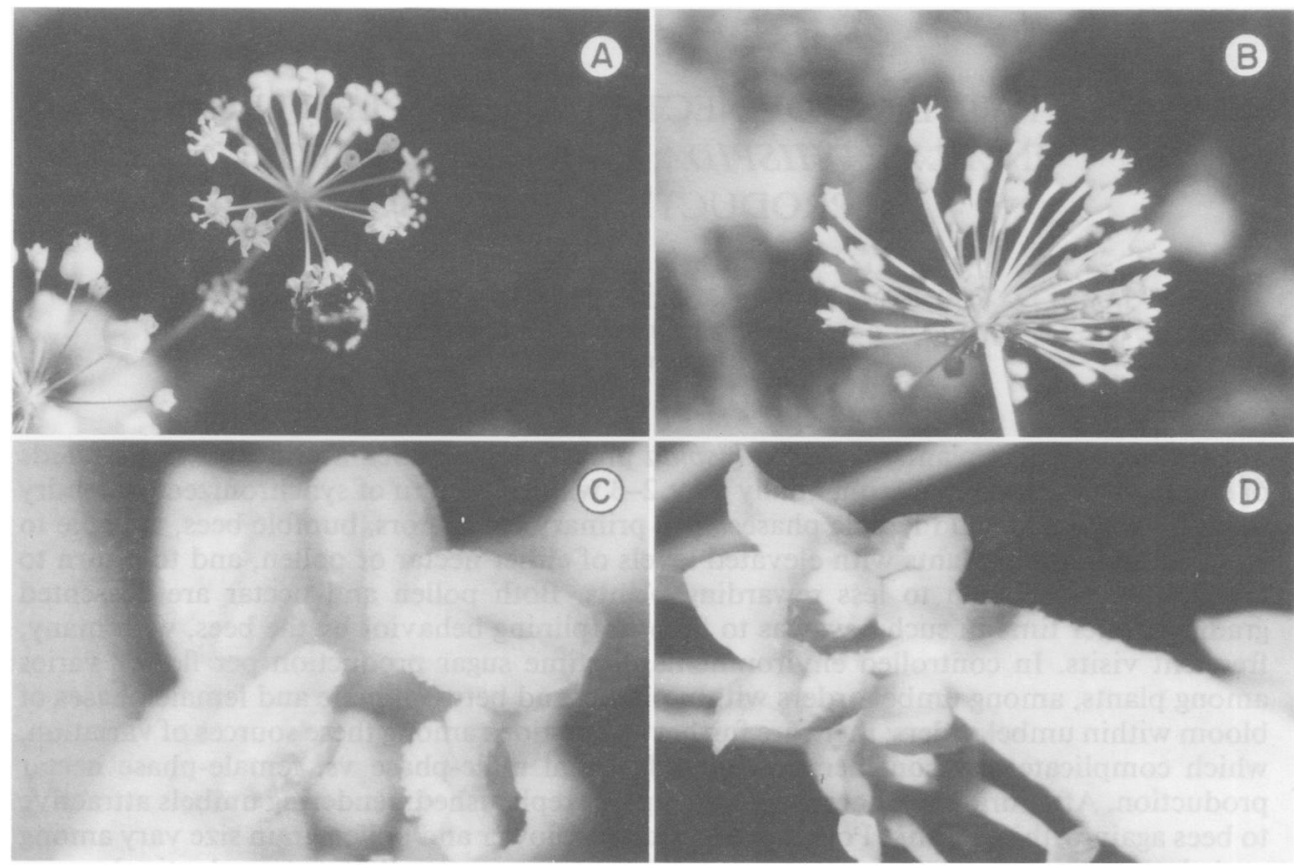

Fig. 1A. (Upper left) An umbel of Aralia hispida in the male phase of bloom. Note that flowers open sequentially and centripetally. The central buds will open and shed pollen.

1B. (Upper right) A female-phase umbel. Petals and anthers have been shed; the central, perfect flowers with diverging styles and slightly swollen ovaries are receptive and secreting nectar, whereas the purely staminate peripheral flowers (whose rudimentary styles remain fused) have no further sexual function.

1C. (Lower left) A male-phase flower showing staggered dehiscence of the five anthers. Note that the two valves of an anther may open at different times.

1D. (Lower right) Blotting nectar from a flower with a filter-paper wick.

ulation of nectar (Thomson 1988) or pollen (Thomson 1989) causes increased visitation to the more rewarding plants. Nectar variation may be more important than pollen variation; at least, in the presence of artificially large interplant differences in nectar, bees are unresponsive to the presence or absence of pollen. The bees' preference for enriched plants is persistent; even after nectar augmentation ceases, visitation remains high for at least several hours (trapline holdover, Thomson 1988). Upon encountering a plant recently drained of nectar, a bee will usually leave the plant after probing 2-3 flowers, but will probe all the flowers on a nectar-enriched plant.

The bees therefore seem to be tracking nectar and pollen resources that vary in a complex spatio-temporal pattern. The general goal of this work was to characterize the temporal aspects of that pattern within plants, and to examine interplant variation. More specific questions were: (1) Do nectar production rates differ between male and female phases, in a manner suggesting sex-role specialization (see Willson 1979, Bawa $1980 a$, Devlin and Stephenson 1985, 1987, Devlin et al. 1987)? (2) Is nectar secretion rapid enough to explain revisitation rates on the order of $10 \mathrm{~min}$ ? (3) How does the temporal pattern of reward production affect plant reproductive success, especially given that pollen serves both reproductive and reward functions?

\section{METHODS \\ General}

We conducted field work in recently logged sites near Wesley and Northfield, Maine, in 1983-1984 (see Thomson and Barrett 1981a, Thomson et al. 1982, 1987). For laboratory studies, we dug plants from the study populations in 1980-1982, potted them in local soil, and maintained them outdoors at Stony Brook, New York. Such plants, brought into the greenhouse in January or later, flowered normally to provide material for laboratory study of nectar and pollen.

Aralia hispida nectar is easily accessible, but is often scanty and viscous, making capillary-tube extraction impractical. We used small wicks of Whatman Number 1 filter paper (cut with an insect-pinning point punch for uniformity) to blot up the available nectar (Fig. 1D). We then air dried the wicks, for later redissolution of sugars and colorimetric determination of total carbohydrate using an anthrone assay (McKenna and Thomson 1988). Because we often resampled flowers, an additional advantage of wicks over capillaries was the reduced likelihood of damage to the nectaries.

We counted and measured pollen with a Coulter TAII particle counter equipped with a $280 \mu \mathrm{m}$ aperture tube and a particle-size channelizer accessory. Typically, we harvested a number of undehisced anthers 
with fine forceps, placed them in a clean 1.5-mL polypropylene centrifuge tube, allowed the anthers to dehisce by air drying, then added $70 \%$ ethanol to preserve the grains until counting was done, several months later. Then the grains were suspended in $100 \mathrm{~mL}$ of $0.1 \% \mathrm{NaCl}$, and three $2.0-\mathrm{mL}$ aliquots counted (in particle size channels 6-11). The mean grain size was calculated as a weighted mean based on the size classes (Harder et al. 1985).

\section{Nectar sugar production-lab study}

In 1984, we brought large flowering plants from the greenhouse into a growth chamber and maintained them at $25^{\circ} \mathrm{C}$ with a $14 / 10 \mathrm{~h}$ light/dark cycle. We marked individual flowers to give a sample of 10 flowers in each of the three umbel orders on each plant. We chose central flowers, i.e., those most likely to be hermaphroditic, so that sugar production in the male and female phases could be compared within flowers. For secondary and tertiary umbel orders, the chosen flowers were stratified over the umbels available. After a flower opened, we blotted its nectar at $\approx 1000$ and 1600 until the end of the male phase, as indicated by the dehiscence of the last anther and the subsequent cessation of nectar production. All the wicks used for one flower were pooled to yield the male-phase sugar production. We resumed blotting when the styles diverged; nectar secretion usually resumes at this stage, after being turned off for 1-5 d. By blotting until secretion stopped again, we obtained a second series of wicks containing the female-phase sugar production of each flower. A few flowers wilted or were broken off during sampling, and others (especially in later umbel orders) turned out to be male-only; all were eliminated from the analysis.

\section{Field studies of nectar refilling rate and bee acceptance thresholds}

On the afternoon of 22 July 1984, a warm sunny day, we observed bumble bee visits to four plants. When we saw a bee visit a large, secondary, male-phase umbel and feed from all its flowers, we noted the time, marked the umbel, and then kept all other visitors away by shooing them off. After a specified time interval ranging from 0 to $40 \mathrm{~min}$, we blotted the nectar from six flowers, and subsequently analyzed the pooled wicks as described above. Some wicks were dropped or contaminated: 7 of the 22 samples were based on five flowers, and two samples were based on four flowers. In all cases, carbohydrates were expressed on a perflower basis.

On 17 July 1984, we followed foraging bumble bees, scoring secondary, male-phase umbels they visited as accepted or rejected. These responses are quite distinct (Thomson et al. 1987): rejection meant that the bee landed, probed either one or two flowers on an umbel with at least 12 open flowers, and then left the plant. After such a visit, we blotted nectar from six of the unvisited flowers. Acceptance meant that the bee fed at all of the open flowers on the first umbel visited, then moved to the adjacent paired umbel, and continued to feed. After the bee had drained 4-6 flowers on the second umbel, we shooed it away and then blotted nectar from six of the unvisited flowers of that umbel.

\section{Lab study of pollen quantity, size, and presentation schedule}

We also harvested undehisced anthers from the plants used in the 1984 lab nectar study. We categorized each flower by sexual form (male-only vs. hermaphrodite) and umbel order (primary, secondary, or tertiary). Within each plant, we pooled as many anthers as possible for each category. In 4 out of 48 samples, the number pooled was $<15$; the mean was 22.4 anthers per sample. The pollen grains in the pooled samples were counted and sized.

We also undertook a very detailed study of the time course of pollen presentation within a single umbel or a potted plant. We positioned a secondary umbel with 32 unopened buds under a dissecting microscope in the lab, using a cool illumination source to avoid undue drying of the flowers. We made hourly censuses during the day (the room was darkened at night), scoring the cumulative number of open flowers and of dehisced anthers. The observations spanned $3 \mathrm{~d}$.

\section{Field study of pollen removal}

In collaboration with L. D. Harder and S. C. Peterson, we screened umbels from bees until several flowers had all five anthers dehisced. We exposed each umbel to a single visit from a Bombus worker, noting which flowers were probed. We collected the anthers from one or more visited flowers for Coulter counting of the remaining pollen. From the same umbel, we harvested the nearest flower with undehisced anthers as a control, allowing the anthers to dehisce in a centrifuge tube for counting, as above. The number of grains removed can be estimated by subtraction. We counted 36 experimental flowers (plus controls) from 19 different plants (L. D. Harder, unpublished manuscript).

\section{RESULTS \\ Nectar production patterns}

Sugar production was usually relatively similar for flowers of the same sex phase within umbels, but highly variable at all higher levels (Fig. 2, Table 1). The apparent chaos in Fig. 2 is reinforced by the significant (plant) $\times($ umbel order $) \times($ sex phase $)$ interaction $(\mathrm{Ta}-$ ble 1), which suggests that different plants present very different patterns of nectar as they pass through the successive phases of bloom. Indeed, through the first and second umbel orders, the plants show no unanimity even with respect to qualitative ups and downs of sugar production, although in all five plants, production increased from the secondary female to the tertiary male phase, then declined again to the tertiary female. In four of five plants, mean secondary female produc- 


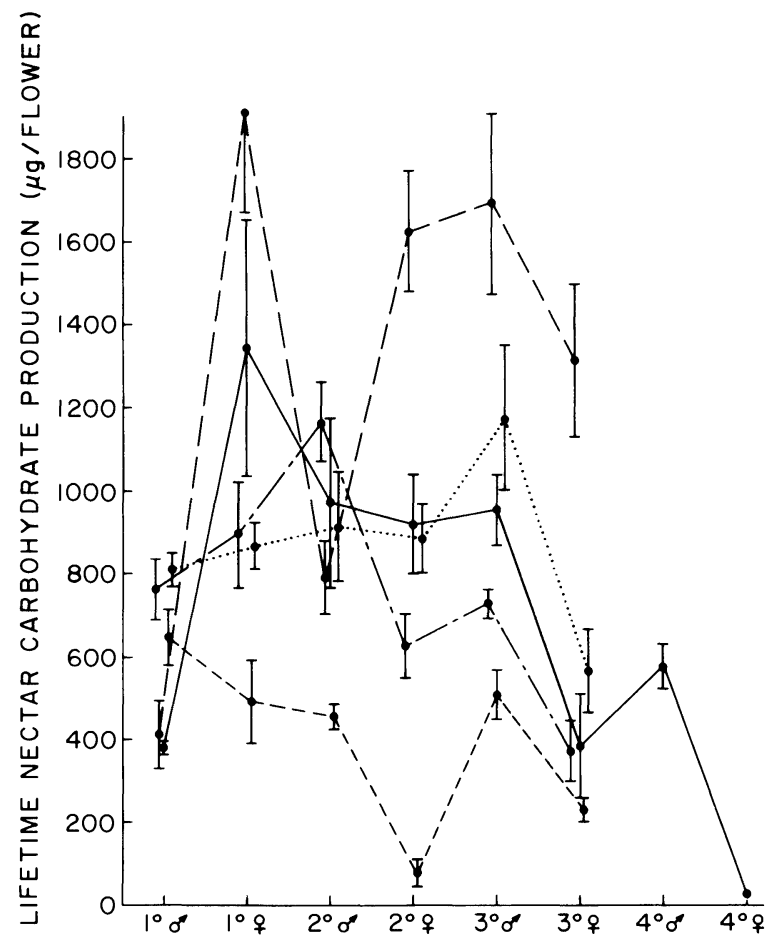

UMBEL ORDER AND SEXUAL PHASE

FIG. 2. Nectar carbohydrate production $(\bar{X} \pm \mathrm{SD})$ through the lifetimes of repeatedly sampled flowers of Aralia hispida, as a function of the temporal sequence of umbel orders and sexual phases. Lines connect points from the same plant.

tion was lower than either of the flanking male phases (secondary male and tertiary male), but in two of these four, the differences were slight. In all plants except number 7, primary female-phase sugar production exceeded that of the preceding primary male phase. Overall, there is no consistent effect of sex phase on sugar production and therefore no indication that either phase is stressing pollinator attraction more than the other.

The strong differences among plants (Fig. 2, Table 1) in overall nectar production may influence natural visitation rates, given the ability of the pollinators to locate and revisit more rewarding plants. However, it is difficult to rule out possible effects of different pot conditions (e.g., slight differences in watering) in producing these effects, although attempts were made to provide uniform culture and growth conditions.

\section{Pollen production and size patterns}

Pollen production per anther varied significantly among umbel orders, increasing from the primary to the tertiary (Table 2). By Tukey studentized range tests, the primary and secondary production figures did not differ significantly, but the tertiary production significantly exceeded that of both preceding umbel orders. There was no difference among plants, and pollen production was equivalent for staminate and perfect flowers within umbels (Table 2).

Plants did differ significantly with respect to mean pollen size (Table 2). Pollen size also increased in later umbel orders, although only the primary and tertiary umbels differed significantly (Tukey studentized range tests; Table 2).

Pollen production and size are positively correlated at the umbel level (Pearson $r=0.45, n=43$ umbels, $P=.003$ ). Although tertiary umbels have fewer flowers than those of previous orders, and although the flowers appear smaller at anthesis, they display a higher absolute investment in male function, having more and larger pollen grains per flower.

\section{Bee responses and refilling rates}

Nectar sugar amounts differed significantly in accepted and rejected umbels (Fig. 3; Mann-Whitney test, $n=11,12, U=121, P<.001)$. There appears to be an acceptance threshold at 10-11 $\mu \mathrm{g}$ carbohydrate per flower, which is far below the lifetime sugar production of a flower (Fig. 2). Nectar is replenished quickly (Fig. 4), and the acceptance threshold can be reached within $15 \mathrm{~min}$.

\section{Pollen presentation by plants}

Within an umbel, flowers open gradually through the day; within a flower, the five anthers open gradually; and within an anther, the two valves often open at different times (Fig. 1C). Under lab conditions, flower openings occur at an almost constant rate during the life of an umbel, and anther dehiscences are even more constant (Fig. 5). In the field, fluctuations of heat and humidity may entrain anthesis and dehiscence so that pollen presentation is more pulsed than in the lab, but is always is gradual.

\section{Pollen removal by bees}

Bombus workers were very effective at stripping dehisced anthers of pollen. Single visits removed $80.1 \%$ (median value) of the grains estimated to be available ( $n=36$; lower quartile $=68.2 \%$, upper quartile $=$ $95.7 \%)$.

TABLE 1. Analysis of nectar production (assayed as total carbohydrate sampled over the entire active phase) by Aralia hispida flowers from five plants, as a function of the umbel order, and the sex phase of the flower (male or female). Analysis of variance by SAS procedure GLM, Type III sums of squares.

\begin{tabular}{|c|c|c|c|c|}
\hline Source of variation & $\mathrm{df}$ & SS & $F$ & $P$ \\
\hline \multicolumn{5}{|l|}{ Model: } \\
\hline Plant & 4 & $1877.06 \times 10^{4}$ & 28.77 & .0001 \\
\hline Umbel order & 2 & $17.87 \times 10^{4}$ & 0.55 & .5790 \\
\hline Sex phase & 1 & $0.18 \times 10^{4}$ & 0.00 & .9734 \\
\hline $\begin{array}{l}\text { Flowers within } \\
\text { plants }\end{array}$ & 45 & $592.63 \times 10^{4}$ & 0.81 & .8002 \\
\hline Plant $\times$ order & 8 & $381.59 \times 10^{4}$ & 2.92 & .0042 \\
\hline Plant $\times$ sex & 4 & $859.71 \times 10^{4}$ & 13.18 & .0001 \\
\hline Order $\times$ sex & 2 & $835.74 \times 10^{4}$ & 25.62 & .0001 \\
\hline \multicolumn{5}{|l|}{ Plant $\times$ order } \\
\hline$\times \operatorname{sex}$ & 8 & $549.36 \times 10^{4}$ & 4.21 & .0001 \\
\hline Error: & 193 & $3148.04 \times 10^{4}$ & & \\
\hline Total (corrected): & 267 & $8555.52 \times 10^{4}$ & & \\
\hline
\end{tabular}


TABLE 2. Pollen production and pollen size in flowers of Aralia hispida.

a. Pollen production and pollen size, categorized by the sexual form of the flower and by the umbel order.

\begin{tabular}{|c|c|c|c|c|c|c|}
\hline \multirow[b]{3}{*}{ Flower sexual form } & \multicolumn{6}{|c|}{ Umbel order } \\
\hline & \multicolumn{2}{|c|}{ Primary } & \multicolumn{2}{|c|}{ Secondary } & \multicolumn{2}{|c|}{ Tertiary } \\
\hline & $\bar{X}$ & (SD) & $\bar{X}$ & (SD) & $\bar{X}$ & (SD) \\
\hline \multicolumn{7}{|l|}{ Staminate } \\
\hline $\begin{array}{l}\text { Pollen production } \\
\text { (grains per anther) }\end{array}$ & 1033 & $(281)$ & 1038 & $(162)$ & 1831 & $(341)$ \\
\hline Pollen grain diameter $(\mu \mathrm{m})$ & 17.5 & $(6.5)$ & 19.6 & $(1.2)$ & 21.2 & $(0.8)$ \\
\hline$n$ & \multicolumn{2}{|c|}{4} & \multicolumn{2}{|c|}{8} & \multicolumn{2}{|c|}{7} \\
\hline \multicolumn{7}{|l|}{ Perfect } \\
\hline $\begin{array}{l}\text { Pollen production } \\
\quad \text { (grains per anther) }\end{array}$ & 713 & $(204)$ & 1268 & $(189)$ & 1941 & $(224)$ \\
\hline Pollen grain diameter $(\mu \mathrm{m})$ & 19.3 & (1.4) & 20.2 & (1.3) & 20.6 & $(0.8)$ \\
\hline$n$ & \multicolumn{2}{|c|}{4} & \multicolumn{2}{|c|}{11} & \multicolumn{2}{|c|}{9} \\
\hline
\end{tabular}

b. Analyses of variance of pollen production and pollen size.*

Source of variation

$\mathrm{df}$

SS

F

$P$

Model:

Plant

Umbel order

Sexual form

Order $x$ form

Error:

Total (corrected):

Model:

Plant

Umbel order

Sexual form

Order $x$ form

Error:

Total (corrected):
Pollen production

$\begin{array}{rll}189.7 \times 10^{4} & 0.90 & .492 \\ 579.7 \times 10^{4} & 6.89 & .003 \\ 3.8 \times 10^{4} & 0.09 & .766 \\ 32.8 \times 10^{4} & 0.39 & .680\end{array}$

$1345.4 \times 10^{4}$

$2294.9 \times 10^{4}$

Pollen size

$\begin{array}{ll}3.14 & .020 \\ 4.19 & .024 \\ 1.63 & .211 \\ 0.88 & .424\end{array}$

* Analysis of variance by SAS procedure GLM, Type III sums of squares.

\section{DisCusSION}

\section{Implications for bee behavior}

In previous studies (Thomson et al. 1982, 1987), we had been struck by how soon a bee would return to a traplined plant. It seemed unlikely that nectar resecretion would be fast enough to make a 10-15 min turnaround time profitable, and we speculated that the frequent revisits might be related to pollen, rather than nectar, foraging, or to shutting out potential competitors. In fact, sufficient nectar accumulates in $15 \mathrm{~min}$ to elicit an acceptance response (Fig. 4). Whether enough sugar has built up for the bee to make an energetic profit is unclear, and it remains possible that the bees may use a threshold amount of nectar simply as a cue to the available pollen. For example, the observed foraging decisions, although empirically shown to be nectar based, could conceivably benefit the bees more through their pollen foraging than through nectar. Even if bees can monitor pollen pickup directly (perhaps by corbicular mechanoreceptors [Ford et al. 1981] or by odors [Dobson 1988]), nectar could still be a better pollen availability cue than pollen itself. Imagine two plants, each with 100 flowers. Plant $A$ has just been thoroughly visited by a bee and has no nectar and no unstripped anthers. Plant B was visited $15 \mathrm{~min}$ ago and has since then accumulated five flowers with freshly dehisced anthers; also, most of its flowers have resecreted nectar. A pollen-hunting bee, using only pollen cues, would need to sample many flowers, on average, to detect the difference between $\mathrm{A}$ and $\mathrm{B}$, whereas a bee using nectar cues would need to probe only a few flowers of either plant to obtain the information needed to predict the presence or absence of available pollen somewhere on the plant.

Given that pollen and nectar supplies are correlated in Aralia hispida, it is obviously difficult to establish which one is ultimately more important to the bee's foraging choices. Several considerations suggest that pollen is important to bees in this situation, however. 


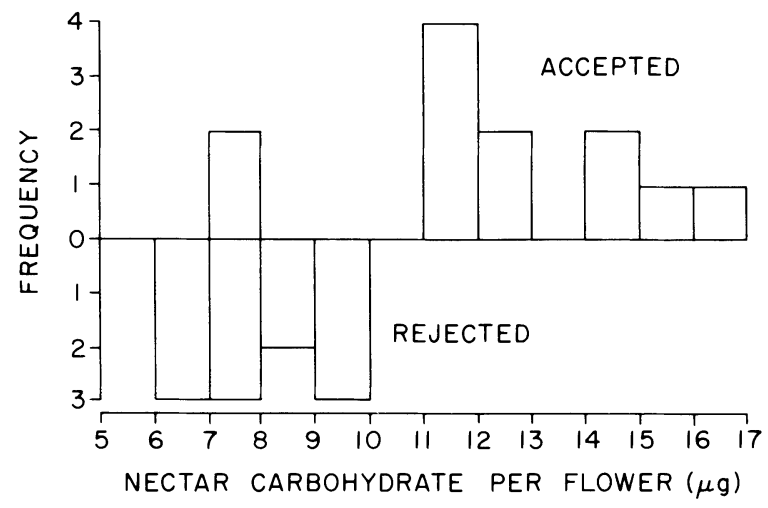

FIG. 3. Distribution of nectar (as carbohydrate per flower) standing crops from unvisited flowers on 23 umbels of Aralia hispida plants. The umbels are categorized by whether a visiting bee accepted or rejected the plant (see Methods: Field Studies of Nectar Refilling Rate and Bee Acceptance Thresholds). The standing crop estimate for each umbel is the mean for six flowers; each umbel sampled was from a different plant.

First, colonies growing in floristically similar habitats in New Brunswick typically fed their entire pollen supplies to larvae during the night, whereas their nectar stores remained ample the next morning (Plowright and Pendrel 1977). Second, the dropping of a plant from a bee's trapline often follows a shift from male to female phase, but seldom the reverse (Thomson et al. 1982). Third, bees prefer polleniferous plants to emasculated plants in some experimental situations (when they are prevented from exercising nectar preferences; Thomson 1989).

Regardless of the relative importance of the two floral rewards, it is clear that the temporal presentation patterns of both make it advantageous for bees to return to the same plants frequently, with consequences for the reproductive success of the plants.

\section{Nectar presentation and plant reproductive success}

The significant interplant differences in sugar production per flower are especially interesting in light of the demonstration that experimental augmentation of nectar increases the visitation rate to individual Aralia hispida inflorescences. Bees apparently discover the locations of more rewarding plants and return there more frequently (Thomson 1988). Those augmentations were much larger than the differences in interplant means reported here, however, so it is not certain that the range of natural variation under field conditions would be sufficient to induce significant interplant difference in visitation rate. Variation in flower number per plant, which is extreme, might well swamp any interplant discrimination based on nectar production differences, especially if the bees are in effect responding to total sugar per plant (i.e., number of flowers $\times$ sugar per flower). However, if the pollinators use nectar-standing-crop threshold as a decision cue for returning to plants as well as for deciding whether to leave or stay, then nectar per flower might have an important influence on visitation rate, independent of flower number variation.

If nectar differences among plants do result in any fitness variation (acting through visitation rate), effects on male reproductive success (RS) should exceed those on female RS. Fruit and seed set are high and uniform in Aralia hispida, with no suggestion of pollinator limitation (Thomson and Barrett 1981a, and personal observations spanning $6 \mathrm{yr}$ at three sites). In such a case, one would expect that pollinator attractants or rewards would have primarily male functions (see Bell 1985, Stanton et al. 1986, Cruzan et al. 1988), and one might further predict that a plant like Aralia hispida, in which male and female functions are separated in time, might secrete more nectar per flower during the male phase (Bawa 1980a, Bullock and Bawa 1981, Devlin and Stephenson 1985, 1987). Although there are hints of such a pattern in the later stages of bloom, there is no such difference overall. However, the hypothesis is too simplistic. First, the trapline holdover phenomenon means that nectar production during one phase could also benefit the RS of the succeeding phase. (The extent of such benefits depends on the length of the holdover, which Thomson's [1988] short experiments are not sufficient to assess.) However, the primary value of female-phase nectar production could be the retention of pollinators to deliver the pollen of the next umbel

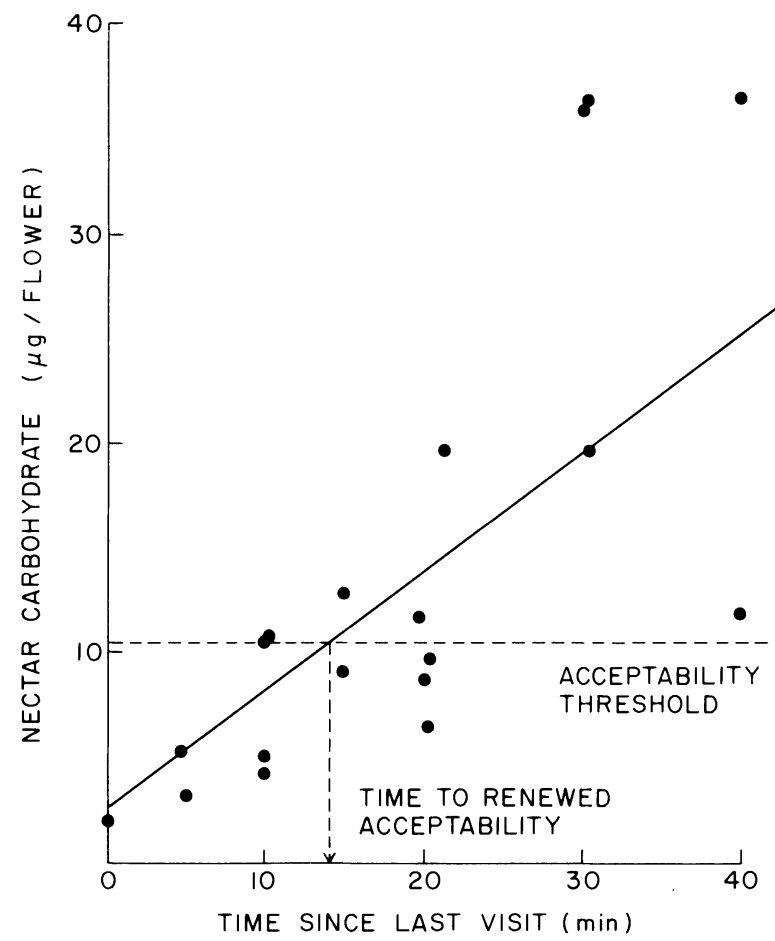

FIG. 4. The rate of nectar refilling (as carbohydrate production) following drainage of Aralia hispida flowers by Bombus workers. — the least squares regression. - - reflection of the empirically-derived acceptability threshold (Fig. 3) by the regression line gives an approximate time to renewed acceptability of $\approx 14 \mathrm{~min}$. 


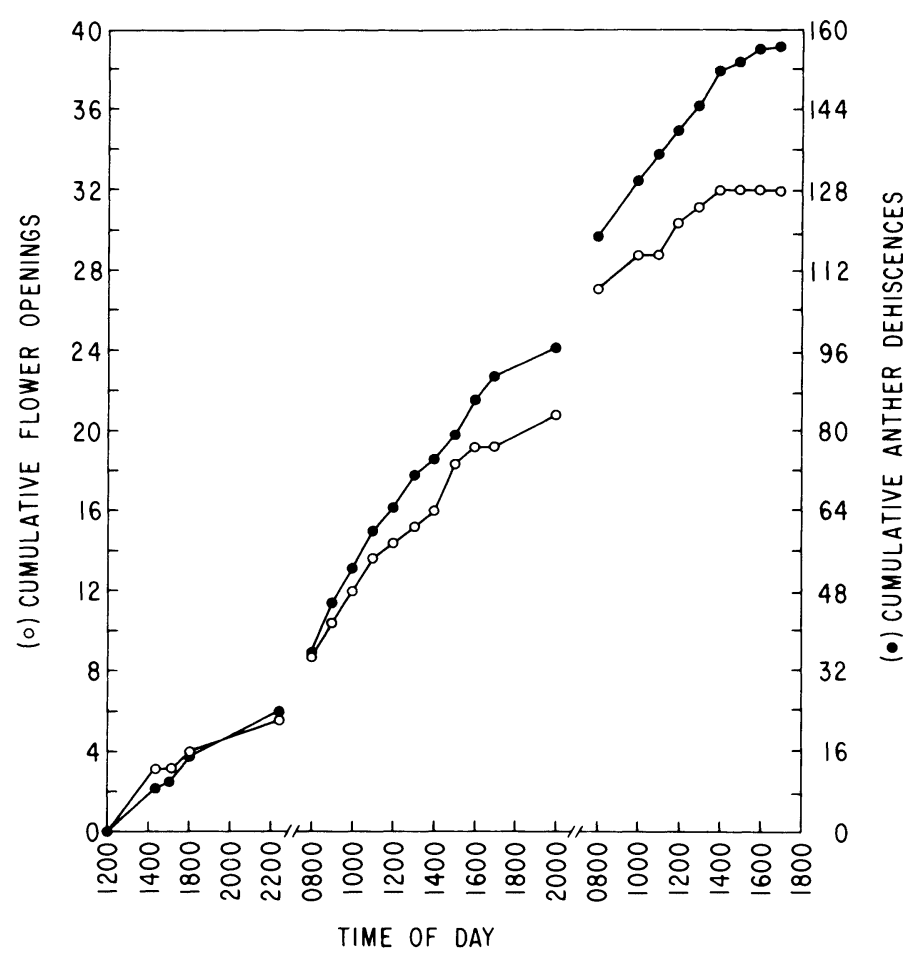

FIG. 5. The schedule of pollen presentation by a single, 32-flowered umbel of Aralia hispida. $\bigcirc$ flower openings, $\bullet$ halfanther dehiscences (see Fig. 1C). The lines break (and the time scale is interrupted) where observations were discontinued overnight.

order. Thus, trapline holdovers further complicate the assignment of specific sex roles to rewards, even when the rewards may be temporally associated with only one of the sexual functions. It may be significant that female-phase sugar production is most consistently lower than male in the tertiary umbels, which usually have no succeeding male phase. Second, sugar per flower is a poor indicator of the phase-specific value of an umbel to a bee, because flower number also varies, being greater in the male phase (Thomson and Barrett $1981 a$ ). Third, the rate of nectar production per unit time is probably higher for male-phase flowers because of their shorter active life-span, although sex-phase longevity is hard to measure because female function diminishes gradually over several days (Thomson and Barrett 1981a). Fourth, although male-phase flowers may not outproduce the female phase in nectar, they do present the additional reward of pollen. Thus, one can defend the conclusion that the male phase of an umbel of $A$. hispida shows more investment in pollinator attraction than the female phase.

\section{Pollen presentation and plant reproductive success}

The increase in pollen production and grain size in later umbel orders was surprising, because later orders produce smaller umbels (fewer flowers, slightly smaller flowers, and shorter pedicels). We interpret this pattern as a temporal shift toward phenotypic maleness within the blooming period of the plant. This shift is rein- forced by the declining fraction of perfect flowers in increasing umbel orders, and by the slightly higher perflower incidence of fruit failure in later orders (Thomson and Barrett 1981a). Temporal shifts in gender can have important consequences for mating success (Devlin and Stephenson 1987), with consequent implications for the evolution of sexual expression (Pellmyr 1987).

As in many other bee-pollinated plants, Aralia hispida pollen has an ambiguous role, serving as both attractant/reward and as the vehicle for the male gametes. The general evolutionary implications of this duality are poorly understood. Considering pollen as a reward, it is apparent that its gradual presentation will act jointly with the rapid replenishment of nectar to encourage rapid revisitation. To understand why a rapid revisitation rate might benefit the plants, it is necessary to consider pollen in its other role. Pollen carry-over is limited in $A$. hispida (Thomson et al. 1982); most of the pollen that a bee removes from a plant is packed into the corbiculae while the bee moves from umbel to umbel, both within and between plants. For a single plant visit, then, the amount of pollen delivered to stigmas is likely to be a decelerating nonlinear function of the amount presented. In such a situation, the plant's male function is most efficiently served by presenting small amounts of pollen to each of numerous visitors (see Lloyd and Yates 1982, Lloyd 1984, Harder and Thomson 1989, Thomson 1988). 
Thomson and Barrett (1981a) previously interpreted the prolonged male phases of $A$. hispida as a bet-hedging strategy for increasing access to females; actually, this mechanism really applies only to spreading male function over several days. Within days, we feel that the maximization of pollen donation efficiency (outlined above) is a much more important consideration, and we interpret the prolonged presentation schedules of pollen and nectar primarily as products of selection on male function. The temporally staggered packaging of pollen-as-male-gametes is also well suited to take advantage of these frequent visits, and may result in a virtually linear relationship between pollen production and delivery (Harder and Thomson 1989). This might be unusual for an insect-pollination system (Lloyd 1984: 298-300), and (following an argument by Charlesworth 1984: 346) could be a contributing factor to the variety of sexual systems observed in Aralia (see also Thomson and Barrett 1981 $a, b$ ), or in small-flowered plants generally (Bawa 1980b, Muenchow 1987).

\section{ACKNOWLEDGMENTS}

We thank L. D. Harder for comments and C. Barton, B. Bingham, J. Guttierez, L. Harder, D. Hensley, J. Hey, K. Krause, M. McKenna, T. McKenna, S. Peterson, D. Ruderman, and B. Thomson for help with the research. This work was funded by EarthWatch and NSF grant DEB 8206959 to J. Thomson and is contribution 687 from Graduate Studies in Ecology and Evolution from the State University of New York at Stony Brook.

\section{LITERATURE CiTED}

Bawa, K. S. $1980 a$. Mimicry of male by female flowers and intrasexual competition for pollinators in Jacaratia dolichaula (D. Smith) Woodson (Caricaceae). Evolution 34: 467-474.

- $1980 \mathrm{~b}$. Evolution of dioecy in flowering plants. Annual Review of Ecology and Systematics 11:15-40.

Bell,G. 1985. On the function of flowers. Proceedings of the Royal Society, London, B, Biological Sciences 224:233265 .

Bullock. S. H., and K. S. Bawa. 1981. Sexual dimorphism and the annual flowering pattern of Jacaratia dolichaula (D. Smith) Woodson (Caricaceae) in a Costa Rican rain forest. Ecology 62:143-150.

Charlesworth, D. 1984. Androdioecy and the evolution of dioecy. Biological Journal of the Linnean Society, London 22:333-346.

Cruzan. M. B., P. R. Neal, and M. F. Willson. 1988. Floral display in Phyla incisa: consequences for male and femalt $\rightarrow$ reproductive success. Evolution 42:505-515.

Devlin, B., J. B. Horton, and A. G. Stephenson. 1987. Patterns of nectar production of Lobelia cardinalis. American Midland Naturalist 117:289-295.

Devlin. B., and A. G. Stephenson. 1985. Sex differential floral longevity, nectar secretion, and pollinator foraging in a protandrous species. American Journal of Botany 72:303310.
Devlin, B., and A. G. Stephenson. 1987. Sexual variations among plants of a perfect-flowered species. American Naturalist 130:199-218.

Dobson. H. E. M. 1988. Survey of pollen and pollenkitt lipids-chemical cues to flower visitors? American Journal of Botany 75:170-182.

Ford, D. M., H. R. Hepburn, F. B. Moseley, and R. J. Rigby. 1981. Displacement sensors in the honeybee pollen basket. Journal of Insect Physiology 27:339-346.

Harder, L. D., and J. D. Thomson. 1989. Evolutionary options for maximizing pollen dispersal of animal-pollinated plants. American Naturalist 133:323-344.

Harder, L. D., J. D. Thomson, M. B. Cruzan, and R. S. Unnasch. 1985. Sexual reproduction and variation in floral morphology in an ephemeral vernal lily, Erythronium americanum. 1985. Oecologia (Berlin) 67:286-291.

Lloyd, D. G. 1984. Gender allocations in outcrossing cosexual plants. Pages 227-300 in R. Dirzo and J. Sarukhan, editors. Perspectives on plant population ecology. Sinauer Associates, Sunderland, Massachusetts, USA.

Lloyd. D. G., and J. Yates. 1982. Intrasexual selection and the segregation of pollen and stigmas in hermaphroditic plants, as exemplified by Wahlenbergia albomarginata (Campanulaceae). Evolution 36:903-913.

McKenna, M., and J. D. Thomson. 1988. A technique for sampling and measuring small amounts of floral nectar. Ecology 69: 1036-1037.

Muenchow, G. 1987. Is dioecy associated with fleshy fruit? American Journal of Botany 74:287-293.

Pellmyr, O. 1987. Multiple sex expressions in Cimicifuga simplex: dichogamy destabilizes hermaphroditism. Biological Journal of the Linnean Society 31:161-174.

Plowright. R. C., and B. Pendrel. 1977. Larval growth in bumblebees (Hymenoptera): (Apidae). Canadian Entomologist 109:967-973.

Stanton, M. L., A. A. Snow, and S. N. Handel. 1986. Floral evolution: attractiveness to pollinators increases male fitness. Science 232:1625-1626.

Thomson, J. D. 1988. Effects of variation in inflorescence size and floral rewards on the visitation rates of traplining pollinators of Aralia hispida. Evolutionary Ecology 2:6576.

Thomson, J. D. 1989. Reversal of apparent feeding preferences of bumble bees by aggression from Vespula wasps. Canadian Journal of Zoology, in press.

Thomson, J. D., and S. C. H. Barrett. 1981a. Temporal variation of gender in Aralia hispida Vent. (Araliaceae). Evolution 35:1094-1107.

Thomson. J. D.. and S. C. H. Barrett. 1981b. Selection for outcrossing. sexual selection, and the evolution of dioecy in plants. American Naturalist 118:443-449.

Thomson. J. D.. W. P. Maddison. and R. C. Plowright. 1982. Behavior of bumble bee pollinators of Aralia hispida Vent. (Araliaceae). Oecologia (Berlin) 54:326-336.

Thomson, J. D., S. C. Peterson, and L. D. Harder. 1987. Response of traplining bumble bees to competition experiments: shifts in feeding location and efficiency. Oecologia (Berlin) 71:295-300

Willson, M. F. 1979. Sexual selection in plants. American Naturalist 113:777-790. 